\title{
Losartan Preserves Erectile Function by Suppression of Apoptosis and Fibrosis of Corpus Cavernosum and Corporal Veno- Occlusive Dysfunction in Diabetic Rats
}

\author{
Wen Ji Li Mingxi Xu Meng Gu Da-chao Zheng Jianhua Guo Zhikang Cai \\ Zhong Wang \\ Department of Urology and Andrology, Ninth People's Hospital, School of Medicine, Shanghai Jiaotong \\ University, Shanghai, China
}

\section{Key Words}

Angiotensin II - Apoptosis - Diabetes - Erectile dysfunction - Fibrosis • Veno-occlusive dysfunction

\begin{abstract}
Background/Aims: Transforming growth factor- $\beta 1$ (TGF- $\beta 1$ ) plays important roles in penile corporal fibrosis and veno-occlusive dysfunction (CVOD). Angiotensin II (Ang II) is critically involved in erectile dysfunction, and blocking of Ang II is more important than inhibition of TGF- $\beta$ in non-penile tissue fibrosis. However, the role of Ang II in corporal fibrosis and CVOD in a diabetic condition has not been investigated. Methods: Diabetic rats were treated with sildenafil or losartan (an Ang II antagonist) alone or in combination. Intracavernosal pressure, dynamic infusion cavernosometry, and histological and molecular alterations of the corpus cavernosum were examined. Results: Diabetic rats exhibited decreases in erectile response, severe CVOD, apoptosis, fibrosis, and activation of the TGF- $\beta 1$ pathway. Treatment with sildenafil had a modest effect on erectile response and an insignificant suppressive effect on CVOD, apoptosis, fibrosis, and the TGF- $\beta 1$ pathway. Although losartan greatly improved the histological and molecular changes and CVOD as compared with sildenafil, its effect on erectile response was low. The combination of sildenafil and losartan had superior effects on these parameters than did either compound alone. Conclusion: Ang II activation may be involved in apoptosis and fibrosis of the corpus cavernosum through Smad and non-Smad pathways, resulting in CVOD and ED. The low efficacy of sildenafil in a diabetic ED rat model was at least partly due to its inadequate effects on apoptosis, fibrosis, and CVOD.
\end{abstract}

W.J. Li and M. Xu contributed equally to this work.

Zhong Wang or Zhikang Cai

KARGER
Department of Urology and Andrology, Ninth People's Hospital, School of Medicine,

Shanghai Jiaotong University, 639 Zhizaoju Road, Shanghai 200011 (China)

Tel. +86 21-2327-1007/+86 21-2327-1008, Fax +86 21-6313-6856, E-Mail

zhongwang2010@sina.com/czk19650228@sina.com 


\section{Cellular Physiology Cell Physiol Biochem 2017;42:333-345 \begin{tabular}{l|l} 
DOI: 10.1159/000477388 & O 2017 The Author(s). Published by S. Karger AG, Basel \\
www.karger.com/cpb
\end{tabular}}

Li et al.: Losartan Preserves Erectile Function by Suppression of Fibrosis and CVOD

\section{Introduction}

The increasing incidence of diabetes in adults is a major global public health problem. The number of adults with diabetes is estimated to rise from 382 million in 2013 to 592 million by 2035 [1]. Diabetes is one of the most common causes of erectile dysfunction (ED), which is reported to occur in $32 \%$ of men with type 1 diabetes and $46 \%$ of men with type 2 diabetes [2]. ED occurs at a younger age in diabetic men than in the general population, and about $50 \%$ of diabetic men present with ED within 10 years of their diagnosis [3]. In addition, diabetic ED is more severe and less responsive to phosphodiesterase type 5 inhibitors (PDE5i), the first-line oral treatment for ED, than is non-diabetic ED [4, 5].

Although multiple mechanisms of diabetes-induced ED have been proposed, corporal veno-occlusive dysfunction (CVOD) has clearly been identified as the mechanism accounting for $66-75 \%$ of ED cases regardless of etiology [6]. CVOD is the inability of the corporal smooth muscle mass to relax sufficiently, which results in inadequate compression of the subtunical veins, a concomitant inability to retain blood within the corpora, and, thus, incomplete tumescence. Therefore, any process that damages the structure and/or function of the corpus cavernosum, such as reduced smooth muscle content and increased collagen deposition, or ultimately corporal fibrosis, will predispose an individual to developing CVOD [7]. Numerous studies have demonstrated that apoptosis plays a critical role in fibrosis in different organs such as the liver, lungs, kidneys, heart, and penile corpora cavernosa [7-10]. Both animal and human studies have demonstrated that angiotensin II (Ang II) is critically involved in promoting apoptosis, collagen deposition, and tissue fibrosis through various mechanisms [10-14]. The major profibrotic mechanisms of Ang II that are responsible for structural changes in non-penile tissues are the transforming growth factor- $\beta 1$ (TGF- $\beta 1$ )dependent and -independent Smad signaling pathways [14,15].

As shown in studies on other organs such as the heart, kidneys, lungs, and liver [16,17], Ang II as a potent fibrotic factor is involved in hypertension- or diabetes-induced structural changes of the corpus cavernosum $[18,19]$. Our previous study $[20]$ showed that suppression of the TGF- $\beta 1 /$ Smad pathway by a TGF- $\beta 1$ antagonist, P144 peptide, had a synergistic effect with the PDE5i sildenafil and significantly increased erectile response, potentially through antifibrotic activity and attenuation of CVOD. Importantly, a recent study revealed that decreased erectile function was accompanied by TGF- $\beta 1 /$ Smad pathway activation in the rat corpus cavernosum following bilateral cavernous nerve injury. Treatment with losartan, an antagonist of the Ang II type 1 receptor, suppressed the TGF- $\beta 1 /$ Smad pathway and preserved erectile function, suggesting that Ang II type 1 receptor antagonism may counteract structural changes in the corpus cavernosum and preserve erectile function [21]. In addition, recent evidence indicates that blocking of Ang II is more important than inhibition of TGF- $\beta$ in non-penile tissue fibrosis [22], and combined therapy with sildenafil and losartan presented a better outcome in terms of functional and structural modification in the cavernous tissue of spontaneously hypertensive rats (SHR) as compared with either of the two drugs alone [23]. However, the role of Ang II in apoptosis and fibrosis of the penile corpora cavernosa and CVOD in a diabetic condition has not been investigated until recently.

The aim of the present study was to investigate whether activation of Ang II and its downstream Smad- and non-Smad-dependent pathways would induce the apoptosis and fibrosis of the penile corpora cavernosa and CVOD seen in diabetes-induced ED, and whether a combination of losartan and sildenafil could synergistically improve erectile response and cavernosal structure in a diabetic rat model.

\section{Materials and Methods}

\section{Experimental animals}

Eight-week-old male Sprague-Dawley rats weighing 250-280 g were randomly divided into five groups ( $\mathrm{n}=8$ in each group): a normal control group, a diabetic group, a losartan (30 mg/kg/day, oral gavage, MSD, 


\section{Cellular Physiology Cell Physiol Biochem 2017;42:333-345 \begin{tabular}{l|l} 
DOI: 10.1159/000477388 & Ond 2017 The Author(s). Published by S. Karger AG, Basel \\
www.karger.com/cpb
\end{tabular}}

Li et al.: Losartan Preserves Erectile Function by Suppression of Fibrosis and CVOD

Hangzhou, China)-treated diabetic group, a sildenafil (20 mg/kg/day, oral gavage, Pfizer, Dalian, China)treated diabetic group, and a combination sildenafil and losartan-treated diabetic group. Diabetes was induced by a single intraperitoneal injection of $50 \mathrm{mg} / \mathrm{kg}$ streptozotocin (STZ, Sigma-Aldrich, Shanghai, China); the control group was treated with vehicle (0.1 M pH 4.5 citrate buffer solution, Sigma-Aldrich). All rats were fasted overnight $(12 \mathrm{~h}) 3$ days after STZ injection, and their blood glucose levels were measured. Animals were considered diabetic if their blood glucose levels were higher than $16.7 \mathrm{mmol} / \mathrm{L}$. Blood glucose levels and body weights after fasting overnight for $12 \mathrm{~h}$ were measured weekly. High blood glucose levels were maintained throughout the experiment. Six weeks after diabetes induction, each group received the designated treatment for 4 weeks. After a 24-h washout period, erectile function was assessed in all groups. During the entire experimental process, two rats were housed in one cage under a $12 \mathrm{~h} \mathrm{light,} 12 \mathrm{~h}$ dark cycle with ad libitum access to standard rat chow and water. The animal experiments were reviewed and approved by the Institutional Animal Care and Use Committee of the Ninth People's Hospital of Shanghai Jiaotong University School of Medicine.

\section{Evaluation of erectile function}

Intracavernous pressure (ICP) in response to electrical stimulation of the cavernous nerve and mean arterial blood pressure (MAP) were used to evaluate erectile function, as previously described [20]. Briefly, the rats were anesthetized with an intraperitoneal injection of $50 \mathrm{mg} / \mathrm{kg}$ pentobarbital, and a 24-gauge angiocatheter was introduced into the carotid artery and connected to a pressure channel to continuously monitor MAP. The corpus cavernosum was exposed and cannulated with a 24-gauge butterfly needle to permit continuous monitoring of ICP. Pressure data were collected and analyzed electronically (PowerLab/8SP, ADInstruments Pty Ltd, Castle Hill, Australia). The erectile response was elicited by electrical stimulation of the cavernous nerve ( $3 \mathrm{~V}$, width $0.2 \mathrm{~ms}$, duration 30 seconds) at several frequencies $(2.5,5,10$, and $20 \mathrm{~Hz})$. Comparisons were made between the maximum increase in ICP (baseline ICP subtracted from maximum ICP) and MAP as well as total erectile response, which was determined by the area under the curve (AUC) from the beginning of stimulation until the ICP returned to baseline or prestimulation pressures.

\section{Dynamic infusion cavernosometry}

After erectile response was evaluated, cavernosometry was performed as previously described $[6,20]$. Briefly, basal ICP was recorded, and $5 \mathrm{mg} / \mathrm{kg}$ papaverine was administered into the corpus cavernosum. The ICP during tumescence was recorded as "ICP after papaverine." Saline was then infused into another corpus cavernosum, and the infusion rate was increased by $0.05 \mathrm{ml} / \mathrm{min}$ every $10 \mathrm{~s}$ until the ICP reached $100 \mathrm{mmHg}$. The "drop rate" was determined by recording the decrease in ICP in the minute directly after the infusion was halted. Thereafter, the middle sections of the corpus cavernosum were maintained overnight in $10 \%$ formaldehyde solution and paraffin-embedded for histological study. The remaining segments of the corpus cavernosum were rapidly frozen in liquid nitrogen and stored at $-80^{\circ} \mathrm{C}$ until processing.

\section{Detection of apoptosis}

Apoptosis was detected by labeling of DNA fragments using the terminal deoxynucleotidyl transferasemediated 2'-deoxyuridine 5'-triphosphate (dUTP) nick end labeling (TUNEL) method, as previously described [5, 24]. Briefly, after deparaffinization and rehydration, $5 \mathrm{~mm}$ thick sections were pretreated with proteinase $\mathrm{k}$ and quenched. The sections were then incubated with a terminal deoxynucleotidyl transferase (TdT) enzyme and incubated with antidigoxigenin-peroxidase conjugate. After staining with diaminobenzidine (DAB) in staining buffer, the slides were counterstained with methyl green. As a negative control, equilibration buffer was substituted for the TdT enzyme reagent. On each slide, five high-power $(\times 400)$ fields were randomly selected and the apoptotic index was calculated as the percentage of apoptotic cells relative to the total number of cells in the given area. Image analysis was performed by the artificial counting method in a blinded fashion using the same standards in all groups. For randomization, microscopic viewing, reading, and counting were performed blindly by covering the label on each slide. After this, the label of each slide was uncovered and data from each slide were assigned to the corresponding group.

\section{Histological and immunohistochemical examinations}

Masson's trichrome staining and immunohistochemical staining were performed as previously described [20]. For immunohistochemical analysis, the Dako EnVision System (Carpinteria, CA, USA) and 


\section{Cellular Physiology Cell Physiol Biochem 2017;42:333-345 \begin{tabular}{l|l} 
DOI: 10.1159/000477388 & O 2017 The Author(s). Published by S. Karger AG, Basel \\
www.karger.com/cpb
\end{tabular}}

Li et al.: Losartan Preserves Erectile Function by Suppression of Fibrosis and CVOD

a smooth muscle alpha-actin primary antibody (a-SMA,1:50, DAKO, Carpinteria, CA, USA) were used. As a negative control, the primary antibody was omitted. For quantitative analysis, two fields in each slide were randomly viewed at $\times 100$ magnification and the ratio of smooth muscle to collagen and the percentage of smooth muscle alpha-actin in a given area were measured using Image Pro Plus 4.5 software (Medica Cybernetics, Silver Spring, MD, USA). Image analysis was performed in a blinded fashion using the same standards in all groups.

Western blot analysis

The corpus cavernosum was homogenized in ice-cold RIPA Lysis and Extraction Buffer (Pierce Biotechnology, Rockford, IL, USA) containing $25 \mathrm{mM}$ Tris $\cdot \mathrm{HCl} \mathrm{pH}$ 7.6, $150 \mathrm{mM} \mathrm{NaCl}$ 1\% NP-40, 1\% sodium deoxycholate, and $0.1 \%$ SDS with a $1 \times$ final concentration of Halt ${ }^{\mathrm{TM}}$ Protease and Phosphatase Inhibitor Cocktail (Pierce Biotechnology) and centrifuged at $14,000 \mathrm{rpm}$ and $4^{\circ} \mathrm{C}$ for $15 \mathrm{~min}$. Western blot analysis was performed as previously described [20]. Briefly, equal amounts of protein extracts $(20-50 \mu \mathrm{g})$ from the corpus cavernosum were electrophoresed on a sodium dodecyl sulfate-polyacrylamide gel and transferred to polyvinyl difluoride membranes. The membranes were blocked with 5\% nonfat dry milk in Tris-buffered saline with Tween 20 (Bio-Rad Laboratories, Hercules, CA, USA) for $1 \mathrm{~h}$ at room temperature, and incubated overnight at $4^{\circ} \mathrm{C}$ with primary antibodies [anti-TGF- $\beta 1$ and anti-myosin phosphatase target subunit 1 (MYPT1) (1:200; Santa Cruz Biotechnology, Santa Cruz, CA, USA), anti-Smad2/3 and anti-phospho-Smad2 at Ser465/467 (1:1,000; Cell Signaling Technology, Beverly, MA, USA), anti-plasminogen activator inhibitor 1 (PAI-1) and Rho kinase-II (ROCK-II) (1:1,000 and 1:2000, respectively; BD Transduction Laboratories, San Diego, CA, USA), and anti-phospho-MYPT1 at Thr696 (1:1,000, Upstate Biotechnology, Lake Placid, NY, USA)]. The membranes were then incubated with the appropriate horseradish peroxidase-linked secondary antibodies (Pierce Biotechnology) for $2 \mathrm{~h}$ at room temperature and visualized using an electrochemiluminescence detection system (Pierce Biotechnology). The Western blotting results were quantified by densitometry using Bio-Rad Quantity One 4.6.2 imaging software (Bio-Rad Laboratories, Hercules, CA, USA).

Measurement of cavernous cGMP and nitrate plus nitrite levels

To measure cGMP levels, the cavernous samples were assayed using a cGMP ELISA kit (Cayman Chemical, Ann Arbor, MI, USA) according to the manufacturer's instructions. cGMP levels are expressed as femtomoles of cGMP per milligram of protein. Nitrate plus nitrite formation was used as an index of NOS activity $[6,25]$. Therefore, nitric oxide synthase (NOS) activity was evaluated by measuring cavernous tissue nitrate plus nitrite formation using a Nitrate/Nitrite Colorimetric Assay kit (Cayman Chemical) according to the manufacturer's instructions.

\section{Statistical analysis}

All results are expressed as means \pm standard deviations. Given the modest sample size, the nonparametric Kruskal-Wallis test and Mann-Whitney U-test were used to determine statistical significance. A P value $<0.05$ was considered statistically significant.

\section{Results}

\section{General characteristics of diabetes}

Compared to control rats, diabetic rats treated with or without losartan or sildenafil showed a significant increase in blood glucose level and a decrease in body weight. Neither treatment with losartan or sildenafil alone nor combination treatment noticeably affected blood glucose level or body weight compared to absence of treatment (Table 1).

\section{Losartan and sildenafil restore erectile response}

The ratio of peak ICP to MAP (ICP/MAP) and the AUC of the ICP traces are widely used to evaluate erectile function. In the present study, electrical stimulation of the cavernous nerve induced a frequency-dependent increase in both percent ICP/MAP and AUC in all experimental groups. The untreated diabetic group had significantly lower ICP/MAP and 
Table 1. Changes of body weight and blood glucose levels in the experimental groups. Data are expressed as mean \pm SD. $\neq \mathrm{P}<0.01$ compared with control group. $\mathrm{N}=8$ in each group

\begin{tabular}{llclc}
\hline \multirow{2}{*}{ Groups } & \multicolumn{2}{c}{ Body weight (g) } & \multicolumn{2}{c}{ Blood glucose (mmol/L) } \\
& Initial & Final & Initial & Final \\
\hline Control & $279.5 \pm 9.7$ & $508.5 \pm 22.6$ & $5.1 \pm 0.8$ & $5.3 \pm 0.7$ \\
DM & $265.3 \pm 10.5$ & $315.3 \pm 23.5 \neq$ & $4.8 \pm 0.6$ & $27.3 \pm 3.8 \neq$ \\
DM + sildenafil & $278.6 \pm 11.8$ & $317.7 \pm 26.3 \neq$ & $5.3 \pm 0.5$ & $26.5 \pm 3.3 \neq$ \\
DM + losartan & $283.3 \pm 10.7$ & $320.2 \pm 25.7 \neq$ & $5.0 \pm 0.7$ & $26.8 \pm 2.5 \neq$ \\
DM + sildenafil + losartan & $268.7 \pm 9.8$ & $338.8 \pm 27.5 \neq$ & $4.7 \pm 0.5$ & $25.6 \pm 2.7 \neq$ \\
\hline
\end{tabular}

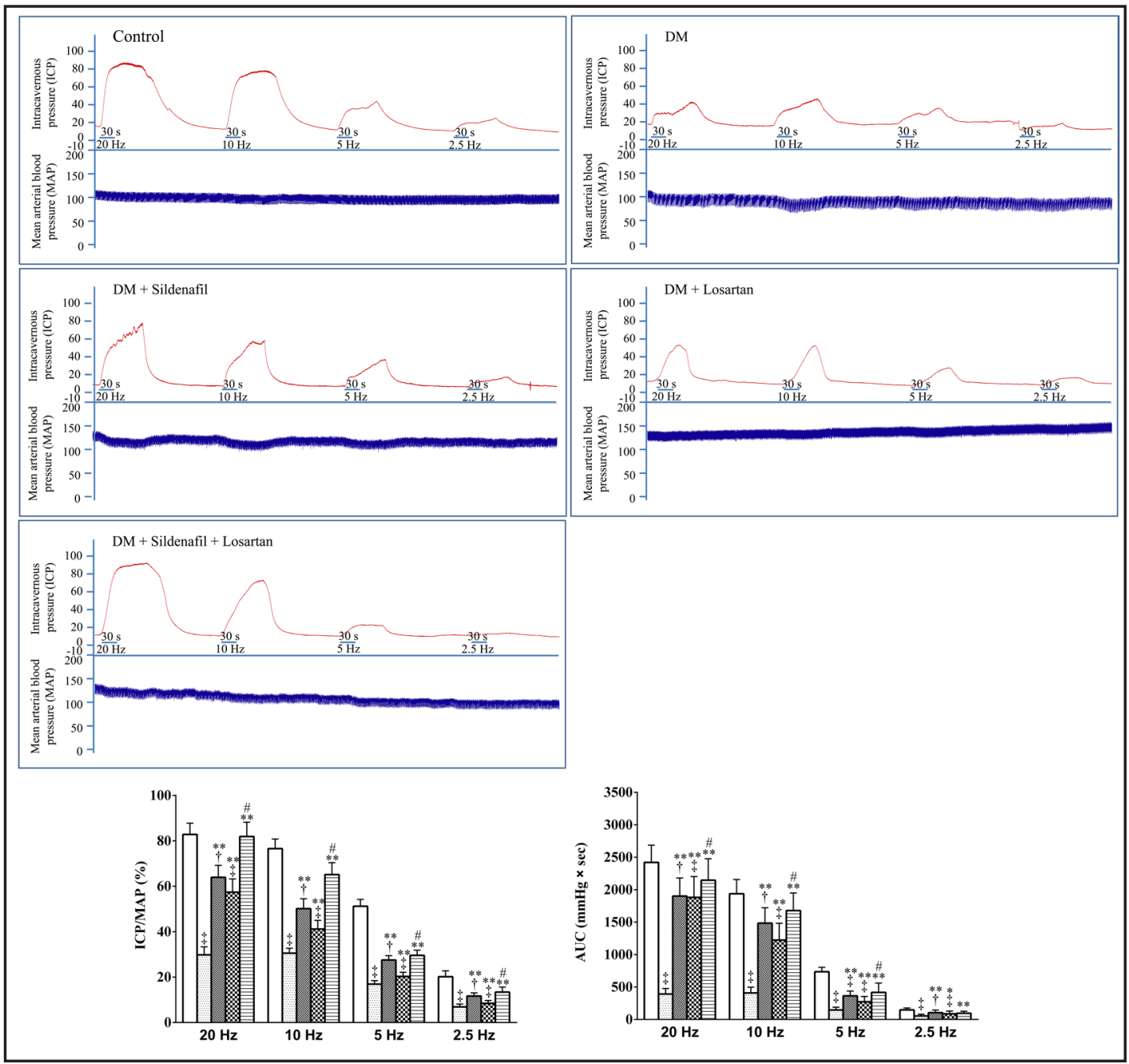

Fig. 1. Evaluation of erectile response. Results of cavernous electrostimulation in the five experimental groups are expressed as percent ICP/MAP and AUC, respectively. Bar graphs represent means \pm SD. $†$ P $<0.05, \neq \mathrm{P}<0.01$ compared with control group; ${ }^{*} \mathrm{P}<0.05,{ }^{* *} \mathrm{P}<0.01$ compared with untreated diabetic group; \# P $<0.05$ compared with losartan-treated diabetic group. DM = diabetes mellitus; ICP = intracavernous pressure; $\mathrm{MAP}=$ mean arterial pressure; $\mathrm{AUC}=$ area under the curve.

AUC values than did the normal control group. Both ICP/MAP and AUC were significantly restored by treatment with losartan or sildenafil alone compared with absence of treatment; however, these parameters were still lower than those in the normal control group, and the 
Fig. 2. Dynamic infusion cavernosometry. Results of dynamic infusion cavernosometry in the five experimental groups are expressed as papaverine response and drop rate. Bar graphs represent means \pm SD. $\dagger$ $\mathrm{P}<0.05, \neq \mathrm{P}<0.01$ compared with control group; * $\mathrm{P}$ $<0.05,{ }^{* *} \mathrm{P}<0.01$ compared with untreated diabetic group; \# $\mathrm{P}<0.05$ compared with sildenafil-treated diabetic group. $\mathrm{DM}=$ diabetes mellitus.
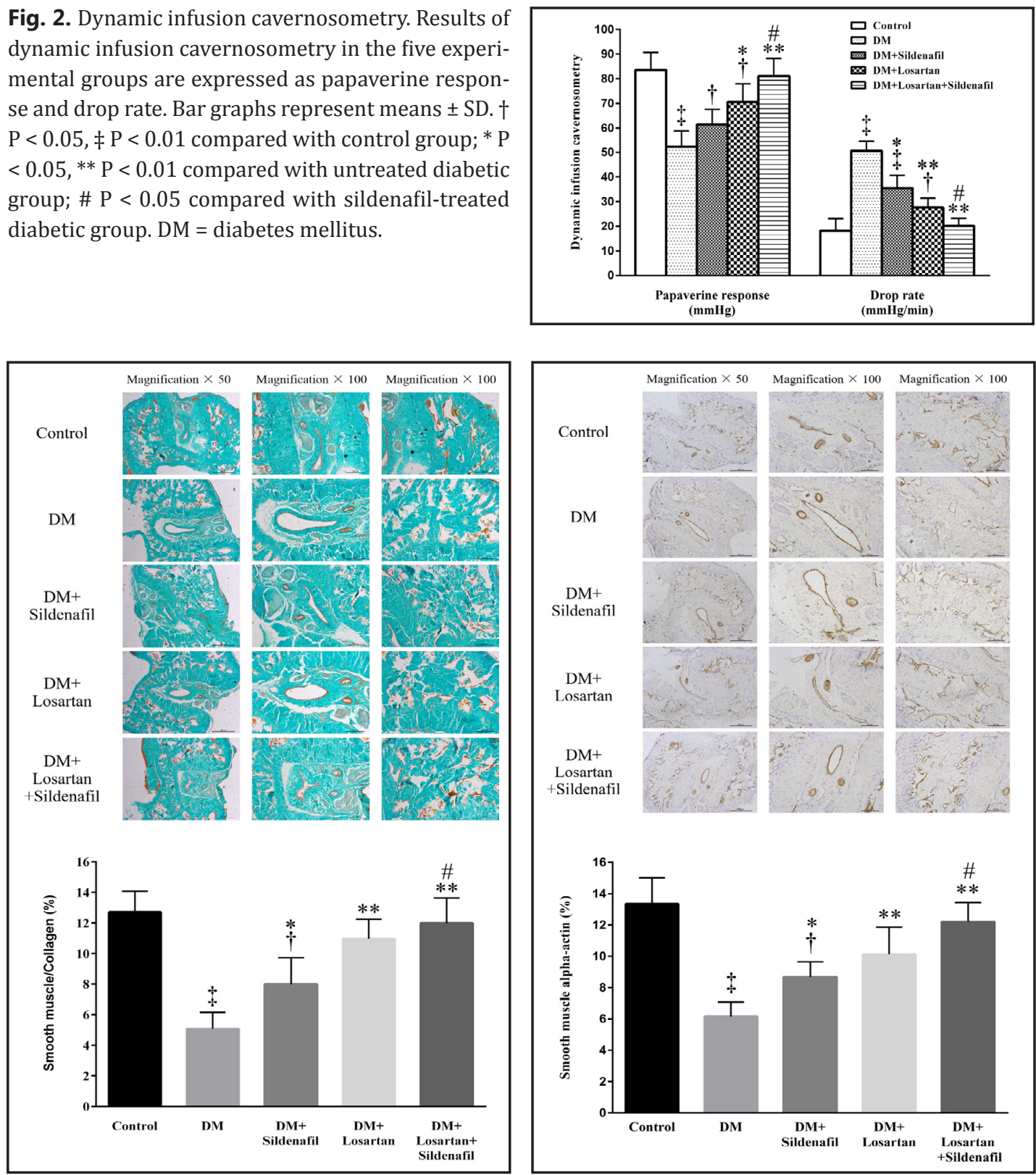

Fig. 3. Masson's trichrome stain. Representative micrographs show smooth muscle component as red color, collagen component as blue color (magnification $\times 100$ ). Bar graphs represent quantitative image analysis. $\dagger \mathrm{P}<0.05, \ddagger \mathrm{P}<0.01$ compared with control group; ${ }^{*} \mathrm{P}<0.05,{ }^{* *} \mathrm{P}<0.01$ compared with untreated diabetic group; \# $\mathrm{P}<0.05$ compared with sildenafil-treated diabetic group. $\mathrm{DM}=$ diabetes mellitus.

Fig. 4. Immunohistochemical stain for smooth muscle alpha-actin. Representative micrographs show penile smooth muscle alpha-actin as brown areas (magnification $\times 100$ ). Bar graphs represent quantitative image analysis. $\dagger \mathrm{P}<0.05, \neq \mathrm{P}<0.01$ compared with control group; $* \mathrm{P}<0.05,{ }^{* *} \mathrm{P}<0.01$ compared with untreated diabetic group; \# P $<0.05$ compared with sildenafil-treated diabetic group. $\mathrm{DM}=$ diabetes mellitus.

erectile effect of losartan was even lower than that of sildenafil. Combination treatment more effectively increased both parameters, and no significant differences were observed in the combination treatment group compared to the normal control group (Fig. 1). 


\section{Cellular Physiology

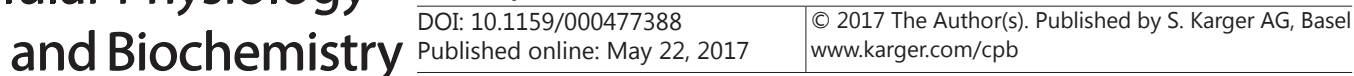 \\ Li et al.: Losartan Preserves Erectile Function by Suppression of Fibrosis and CVOD}

Fig. 5. Detection of apoptosis. Representative micrographs show apoptotic cells as black-brown dots (magnification $\times 400$ ). Bar graphs represent quantitative image analysis. $\uparrow \mathrm{P}<0.05, \neq \mathrm{P}<0.01$ compared with control group; ${ }^{*} \mathrm{P}<0.05$, $^{* *} \mathrm{P}<0.01$ compared with untreated diabetic group; \# $\mathrm{P}<0.05$ compared with sildenafil-treated diabetic group. $\mathrm{DM}=$ diabetes mellitus.

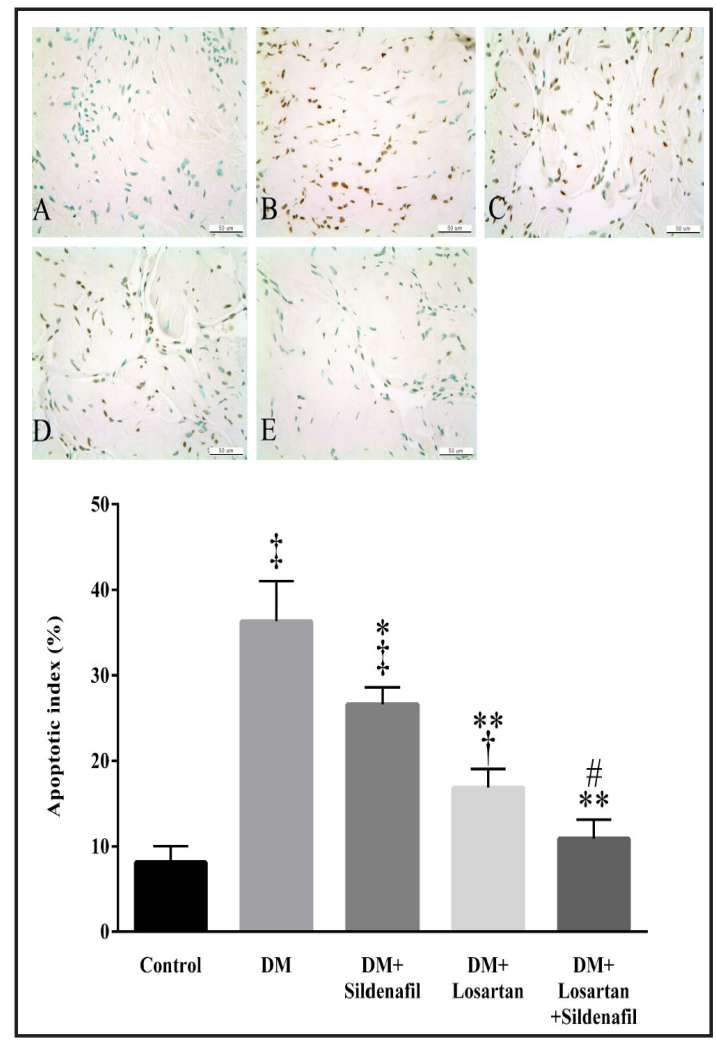

Losartan and sildenafil attenuate CVOD

Dynamic infusion cavernosometry is the standard method used to diagnose CVOD in patients with ED and has been widely used in animal experiments to assess the status of venous leakage $[6,26]$. In the present study, untreated diabetic rats showed a significant decrease in papaverine-induced ICP and an increase in the drop rate compared to those in the normal control group. Rats treated with losartan showed significant attenuation of the decrease in papaverine-induced ICP and the increase in the drop rate compared with untreated diabetic rats, though these parameters were not restored to normal control levels. In contrast to the results of the erectile response tests, the beneficial effect of sildenafil on dynamic infusion cavernosometry was lower than that of losartan. Both the response to papaverine and the drop rate were virtually normalized by combination treatment (Fig. 2).

Losartan and sildenafil restore the smooth muscle/collagen ratio and smooth muscle content in the corpus cavernosum

There was a significant decrease in the smooth muscle/collagen ratio and smooth muscle content in untreated diabetic rats compared with the normal control group. Although neither of the parameters reached normal control levels in losartan-treated diabetic rats, no significant difference was observed between the two groups. Sildenafil also significantly improved these parameters, but the effect was less than that of losartan. Combined treatment exhibited a synergistic effect on these parameters (Fig. 3 and 4).

Losartan and sildenafil reduce diabetes-induced apoptosis in the corpus cavernosum

There was a significant increase in the number of TUNEL-positive cells in the untreated diabetic rats compared to the normal control rats. Although the number of TUNEL-positive cells did not reach control levels, there was no significant difference between the losartantreated group and the normal control group. Sildenafil also significantly improved this parameter, but the effect was less than that of losartan. Combined treatment exhibited a synergistic effect (Fig. 5).

\section{KARGER}




\section{Cellular Physiology and Biochemistry Published \begin{tabular}{l|l} 
DOI: 10.1159/000477388 & $\begin{array}{l}\text { (c) } 2017 \text { The Author(s). Published by S. Karger AG, Basel } \\
\text { www.karger.com/cpb }\end{array}$
\end{tabular} \\ Li et al.: Losartan Preserves Erectile Function by Suppression of Fibrosis and CVOD}

Fig. 6. Western blot analysis. Representative western blots and bar graphs show the expression and densitometric ratios of TGF- $\beta 1$, PAI-1 and ROCK-II to $\beta$-actin, phosphorylated Smad2 to total Smad2, phosphorylated MYPT1 to total MYPT1 in the control rats (lane 1), diabetic rats (lane 2), sildenafil-treated diabetic rats (lane 3 ), losartan-treated diabetic rats (lane 4) and combination of sildenafil and losartan-treated diabetic rats (lane 5) groups, respectively. $\uparrow \mathrm{P}<$ $0.05, \neq \mathrm{P}<0.01$ compared with control group; * $\mathrm{P}<0.05$, ** $\mathrm{P}<0.01$ compared with untreated diabetic group; \# P $<0.05$, \#\# $\mathrm{P}<0.01$ compared with sildenafil-treated diabetic group. TGF- $\beta 1$ = transforming growth factor- $\beta 1$; PAI1 = plasminogen activator inhibitor 1 ; ROCK-II = Rho kinase-II; MYPT1 = myosin phosphatase target subunit 1. DM = diabetes mellitus.

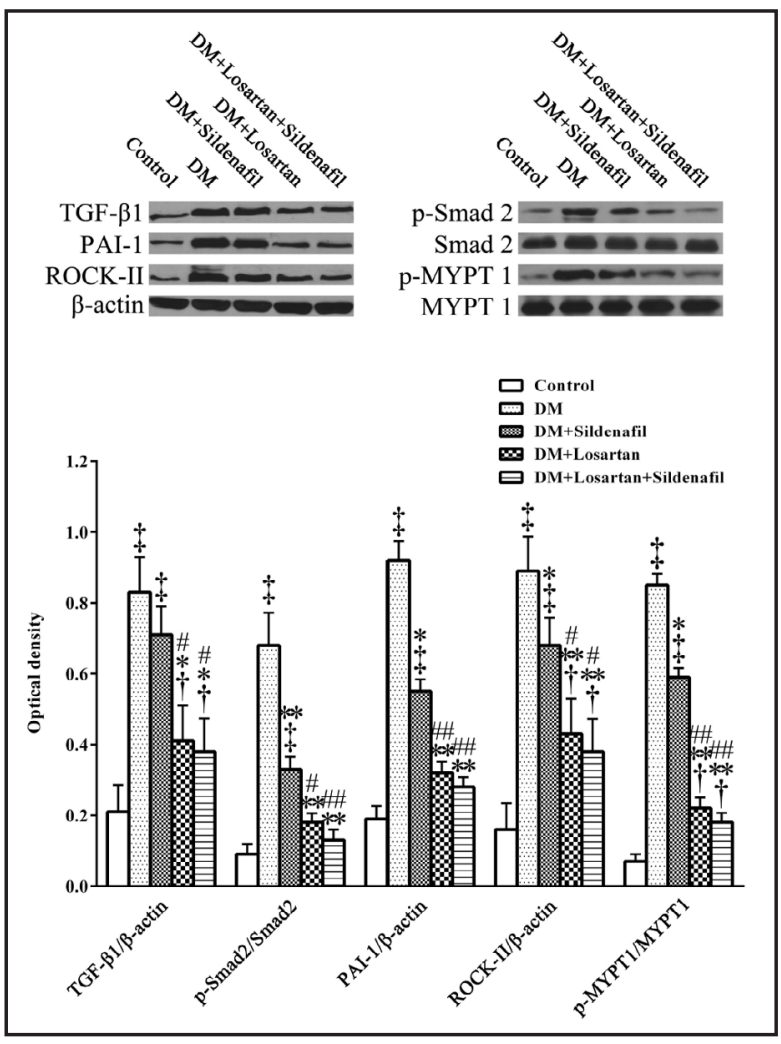

Losartan but not sildenafil suppresses the diabetes-induced increase in TGF- $\beta 1$ expression

The expression of TGF- $\beta 1$ was significantly increased in untreated diabetic rats compared to the normal control group. This increase was significantly inhibited by treatment with losartan. However, treatment with sildenafil did not show a significant suppressive effect on TGF- $\beta 1$ expression compared to that seen in untreated diabetic rats (Fig. 6).

Losartan and sildenafil inhibited diabetes-induced activation of the Smad signaling pathway

Although no significant difference in the total amount of Smad2 was observed among the five groups, diabetic rats showed significantly higher Smad 2 phosphorylation than did normal control rats. Treatment with sildenafil or losartan alone significantly suppressed Smad2 phosphorylation compared to that seen in untreated diabetic rats. However, treatment with losartan more significantly suppressed Smad2 phosphorylation than did treatment with sildenafil, and there was no statistically significant difference between the losartantreated group and the normal control group. Combination treatment with both sildenafil and losartan exhibited a synergistic suppressive effect on Smad2 phosphorylation (Fig. 6). The expression of PAI-1 in untreated diabetic rats increased significantly compared to that in the normal control group, and was significantly reduced by treatment with sildenafil or losartan. However, treatment with losartan more significantly suppressed PAI-1 expression than did treatment with sildenafil, and no statistically significant difference was observed between the losartan-treated group and the normal control group. Combination treatment with both sildenafil and losartan exhibited a synergistic suppressive effect on PAI-1 expression (Fig. 6).

Losartan and sildenafil inhibit diabetes-induced activation of non-Smad-related signaling ROCK-II expression and phosphorylation of MYPT1 at Thr696, an indicator of ROCKII activity, significantly increased in the untreated diabetic rats compared to the normal control group, and decreased significantly following treatment with sildenafil or losartan. The suppressive effect of losartan on these molecules was greater than that of sildenafil. 


\section{Cellular Physiology Cell Physiol Biochem 2017;42:333-345 \begin{tabular}{ll|l} 
DOI: 10.1159/000477388 & $\begin{array}{l}\text { O } 2017 \text { The Author(s). Published by S. Karger AG, Basel } \\
\text { www.karger.com/cpb }\end{array}$
\end{tabular} \\ Li et al.: Losartan Preserves Erectile Function by Suppression of Fibrosis and CVOD}

Table 2. Measurement of NO and cGMP levels. Data are expressed as mean \pm SD. $\dagger \mathrm{P}<$ $0.05, \ddagger \mathrm{P}<0.01$ compared with the control group; ${ }^{* *} \mathrm{P}<0.01$ compared with the untreated diabetic group

\begin{tabular}{lcc}
\multicolumn{1}{c}{ Groups } & $\begin{array}{c}\text { NO (nitrate plus nitrite) } \\
\text { (umol/mg protein) }\end{array}$ & cGMP (fmol/mg protein) \\
\hline Control & $0.78 \pm 0.13$ & $61.35 \pm 4.55$ \\
DM & $0.28 \pm 0.06 \neq$ & $25.35 \pm 2.56 \ddagger$ \\
DM + sildenafil & $0.55 \pm 0.11 \dagger^{* *}$ & $47.25 \pm 3.81 \dagger^{* *}$ \\
DM + losartan & $0.47 \pm 0.09 \dagger^{* *}$ & $38.25 \pm 3.58 \dagger^{* *}$ \\
DM + sildenafil + losartan & $0.72 \pm 0.11^{* *}$ & $59.61 \pm 4.32^{* *}$ \\
\hline
\end{tabular}

Combination treatment with both sildenafil and losartan exhibited a synergistic suppressive effect on expression of these proteins (Fig. 6).

Losartan and sildenafil increase cGMP and nitrate plus nitrite levels

Cavernous NO generation (nitrate plus nitrite) and cGMP levels in the untreated diabetic rats were significantly lower than those in the normal control group. Although treatment with losartan or sildenafil significantly increased NO and cGMP levels, the effect of losartan was less than that of sildenafil. Combination treatment with both sildenafil and losartan had a synergistic effect on NO and cGMP levels (Table 2).

\section{Discussion}

The main finding of the present study is that Ang II plays a critical role in diabetes-induced apoptosis and fibrosis in the corpus cavernosum and severe CVOD via Smad and non-Smad signaling pathways, resulting in an impaired erectile response. In addition, as we described in another study [20], the lower efficacy of the PDE5i sildenafil on diabetes-associated ED vs. non-diabetic ED is due, at least in part, to an insufficient effect on antifibrosis and corporal veno-occlusive function, resulting in venous leakage. Furthermore, the present study further confirmed that to achieve the best therapeutic effect for diabetic ED, it is most important to increase the inflow of blood and decrease venous outflow by simultaneously promoting cavernous smooth muscle relaxation and maintaining structural integrity [20].

Ang II exists in many organs and tissues, including the corpus cavernosum. The human corpus cavernosum produces and secretes Ang II and has a concentration about 200 -fold as high as that in systemic plasma [27]. It has been demonstrated that Ang II plays a critical role in the pathogenesis of ED. In anesthetized dogs, intracavernous injection of Ang II caused termination of spontaneous erection, whereas administration of losartan resulted in smooth muscle relaxation and erection [27]. Also, in human corpus cavernous smooth muscle strips, administration of losartan mediated corpus cavernous smooth muscle relaxation [28]. In addition, the concentration of Ang II was found to be significantly increased in cavernosal tissue in a diabetic rat model, and to markedly reduce the erectile response [29]. Similar to the results from other animal and human studies [29-31], the Ang II type 1 receptor antagonist losartan had a beneficial effect on impaired erectile response in our study. It has been demonstrated that treatment with losartan significantly increases the expression of endothelial nitric oxide synthase in cavernous tissue from SHR [23]. In the present study, an improved erectile response was accompanied by an increase in NO and cGMP levels in the losartan-treated diabetic group, suggesting that overactivation of Ang II may be involved in the impaired erectile response in diabetic rats via suppression of the NO/cGMP pathway.

In addition to suppression of the NO/cGMP pathway, the Ang II-induced impaired erectile response appears to result from apoptosis and fibrosis of the corpus cavernosum and severe CVOD. Diabetes is a well-known etiology of cavernosal fibrosis and CVOD in 
humans and animals [32, 33]. In addition, previous studies have shown that apoptosis in the corpus cavernosum is one of the critical contributors to diabetes-induced ED in diabetic rodents [32, 34]. In the present study, a reduced erectile response was strongly associated with apoptosis and fibrosis in the corpus cavernosum and severe CVOD. After treatment with losartan, apoptosis and fibrosis in the cavernosal tissue and CVOD were attenuated, and the erectile response was significantly improved. These results suggest that Ang II antagonism may play a critical role in the erectile response by improving the structural integrity of the corpus cavernosum and corporal veno-occlusive function. However, treatment with losartan seems to play different roles depending on disease model and animal species. Many studies have found that an impaired erectile response is associated with a significant decrease in smooth muscle content in the corpus cavernosum of diabetic rats [35-37]. Nevertheless, a decrease in erectile function is accompanied by a marked increase in the amount of cavernous smooth muscle from SHR [23]. Treatment with losartan could reduce the amount of cavernous smooth muscle and improve erectile function in SHR [23]. Therefore, further studies are needed to clarify the role of Ang II antagonism in different animal species and different disease models. In addition, Ang II-induced changes in collagen deposition and a-SMA expression in the corpus cavernosum are different from those seen in other organs. In studies on other organs such as the lungs, liver, and heart, Ang II caused increases in both collagen deposition and a-SMA expression. a-SMA, a typical isoform found in smooth muscle cells (SMCs), is not only widely used as a marker of SMCs, but also often used as a marker of myofibroblasts because it is expressed de novo in a-SMA stress fibers [38]. However, a-SMA is non-specific and researchers currently lack a unique set of biomarkers to define myofibroblasts [39]. Even though both SMCs and myofibroblasts express $\alpha$-SMA, their contractile activities are distinct. SMC contractions are reversible and short-lived, whereas myofibroblasts generate isometric contractions that are irreversible and long-lived [39]. Because lung, liver, and cardiac tissues have few SMCs, changes in a-SMA expression levels may represent changes in myofibroblasts that occur during the process of fibrosis. However, the corpus cavernosum contains abundant smooth muscle cells. Therefore, changes in a-SMA expression in the corpus cavernosum may represent changes to SMCs rather than myofibroblasts. Although a-SMA has widely been used as a marker of SMCs in the corpus cavernosum $[6,35,36]$, it is not clear if the regulatory effects of Ang II on collagen deposition and a-SMA expression in the corpus cavernosum are different from those in other organs. Further studies are needed to clarify these issues.

Although apoptosis and fibrosis in the corpus cavernosum and severe CVOD are generally recognized as the pathogenesis of diabetes-associated ED, the underlying pathogenic mechanisms are not fully understood. It has been demonstrated that activation of Ang II is involved in apoptosis of various cells, such as glomerular cells, endothelial cells, podocytes, and cardiomyocytes, in several human diseases and experimental models [4043]. Activation of Smad- and non-Smad-dependent TGF- $\beta 1$ signaling pathways is believed to contribute to Ang II-induced apoptosis and tissue fibrosis [14, 15, 40, 43, 44]. It was previously reported that treatment with losartan suppressed the TGF- $\beta 1 /$ Smad pathway and preserved erectile function [7, 21]. However, these results did not predict whether Ang II antagonism would attenuate apoptosis and fibrosis in the corpus cavernosum and severe CVOD. In the present study, treatment with losartan not only suppressed the TGF- $\beta 1 / \mathrm{Smad} /$ PAI-1 signaling pathway, but also significantly suppressed expression and activity of ROCK in diabetic cavernous tissue. These results may provide clues for a better understanding of the molecular mechanisms of Ang II in apoptosis and fibrosis in the corpus cavernosum and severe CVOD, and of the critical role of Ang II in functional and morphological modification in the cavernous tissue of diabetic rats through Smad- and non-Smad-related signaling pathways. Furthermore, considering the fact that Rho/ROCK plays a pivotal role in diabetic ED by inducing cavernosal fibrosis [45] and increasing smooth muscle tone in the corpus cavernosum [46], Rho/ROCK may also be involved in mediating Ang II-induced diabetesassociated ED. Further studies are needed to clarify this relationship and whether combined 


\section{Cellular Physiology Cell Physiol Biochem 2017;42:333-345

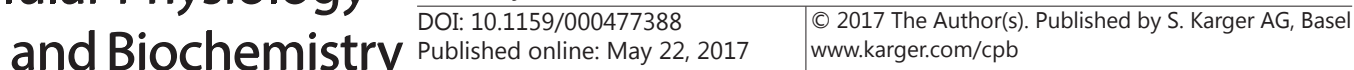 \\ Li et al.: Losartan Preserves Erectile Function by Suppression of Fibrosis and CVOD}

inhibition of both signaling pathways could improve diabetes-induced cavernosal fibrosis and CVOD.

An inadequate effect of sildenafil on the erectile response was strongly associated with relatively small anti-apoptotic and anti-fibrotic effects and attenuation of CVOD as measured by histological examination and dynamic infusion cavernosometry in the present study, which is consistent with our previous report [20]. But, interestingly, the effect of losartan on attenuating apoptosis, fibrosis and CVOD was generally stronger than that of sildenafil. However, its effect on erectile response was relatively weak, as was its impact on NO and cGMP levels. These results suggest that the NO/cGMP pathway may play a more important role in initiating penile erection than in decreasing venous outflow. When combined treatment with both drugs was administered, the erectile response was restored to normal levels. Together with the results of our previous study [20], our current results imply that increasing inflow of blood into the corpus cavernosum or suppressing venous leakage alone is insufficient to achieve a full erection in diabetic conditions. To achieve the best therapeutic effect for diabetic ED, it is therefore most important to increase the inflow of blood and decrease venous outflow by simultaneously promoting cavernous smooth muscle relaxation and maintaining structural integrity [20].

Although the present study is of clinical importance, it has some limitations. We did not compare the harmful effects of Ang II and TGF- $\beta 1$ on cavernosal fibrosis and CVOD. In the current study, losartan but not sildenafil markedly suppressed the expression of TGF- $\beta 1$. As Ang II acts upstream of TGF- $\beta 1$ to directly or indirectly regulate the Smad signaling pathway [22], further studies are needed to clarify the relationship between Ang II and TGF- $\beta 1$ in diabetic cavernous tissue. It is most clinically relevant to establish more potential therapeutic targets for the treatment of diabetes-related ED. In addition, although the STZ-induced diabetic rat model has been used in numerous studies to investigate mechanisms involved in diabetic ED, it is known to mimic type 1 diabetes. Further study should investigate the role of Ang II in type 2 diabetes, which is the predominant form of diabetes.

In conclusion, overactivation of Ang II plays a critical role in diabetes-induced apoptosis and fibrosis in the corpus cavernosum and severe CVOD through Smad- and non-Smaddependent pathways, resulting in impaired erectile response. The reduced efficacy of sildenafil on diabetic ED compared to non-diabetic ED is at least partly due to an inadequate suppressive effect on the molecular alterations that take place during fibrosis and CVOD. The present results contribute to our understanding about the pathophysiology and molecular mechanisms of diabetes-induced ED, and thus help to develop new strategies for clinical treatment of the condition. To achieve the best therapeutic effect for diabetic ED, it is most important to increase the inflow of blood and decrease venous outflow by simultaneously promoting cavernous smooth muscle relaxation and maintaining structural integrity.

\section{Acknowledgements}

This study was sponsored by grants from the Natural Science Foundation of Science and Technology Commission of Shanghai Municipality (No. 12ZR1416700), Shanghai Pujiang Program (No. 13PJD020) and the Natural Science Foundation of China (No. 81172450).

\section{Disclosure Statement}

The authors declare that they have no conflict of interest.

\section{References}

- Guariguata L, Whiting DR, Hambleton I, Beagley J, Linnenkamp U, Shaw JE: Global estimates of diabetes prevalence for 2013 and projections for 2035. Diabetes Res Clin Pract 2014;103:137-149. 


\section{Cellular Physiology Cell Physiol Biochem 2017;42:333-345 \begin{tabular}{l|l|l} 
and Biochemistry 10.1159/000477388 & $\begin{array}{l}\text { D } 2017 \text { The Author(s). Published by S. Karger AG, Basel } \\
\text { www.karger.com/cpb }\end{array}$
\end{tabular}}

Li et al.: Losartan Preserves Erectile Function by Suppression of Fibrosis and CVOD

2 Vickers MA, Wright EA: Erectile dysfunction in the patient with diabetes mellitus. Am J Manag Care 2004;10:S3-11.

3 Thorve VS, Kshirsagar AD, Vyawahare NS, Joshi VS, Ingale KG, Mohite RJ: Diabetes-induced erectile dysfunction: epidemiology, pathophysiology and management. J Diabetes Complications 2011;25:129-136.

4 Penson DF, Latini DM, Lubeck DP, Wallace KL, Henning JM, Lue TF: Do impotent men with diabetes have more severe erectile dysfunction and worse quality of life than the general population of impotent patients? Results from the Exploratory Comprehensive Evaluation of Erectile Dysfunction (ExCEED) database. Diabetes care 2003;26:1093-1099.

-5 Vickers MA, Satyanarayana R: Phosphodiesterase type 5 inhibitors for the treatment of erectile dysfunction in patients with diabetes mellitus. Int J Impot Res 2002;14:466-471.

-6 Ferrini MG, Kovanecz I, Sanchez S, Vernet D, Davila HH, Rajfer J, Gonzalez-Cadavid NF: Long-term continuous treatment with sildenafil ameliorates aging-related erectile dysfunction and the underlying corporal fibrosis in the rat. Biol Reprod 2007;76:915-923.

7 El-Sakka AI, Yassin AA: Amelioration of penile fibrosis: myth or reality. J Androl 2010;31:324-335.

8 Johnson A, DiPietro LA: Apoptosis and angiogenesis: an evolving mechanism for fibrosis. FASEB J 2013;27:3893-3901.

-9 Munoz-Felix JM, Gonzalez-Nunez M, Lopez-Novoa JM: ALK1-Smad1/5 signaling pathway in fibrosis development: friend or foe? Cytokine Growth Factor Rev 2013;24:523-537.

10 Ludwig M, Tolk A, Skorska A, Maschmeier C, Gaebel R, Lux CA, Steinhoff G, David R: Exploiting AT2R to Improve CD117 Stem Cell Function In Vitro and In Vivo--Perspectives for Cardiac Stem Cell Therapy. Cell Physiol Biochem 2015;37:77-93.

-11 Macconi D, Remuzzi G, Benigni A: Key fibrogenic mediators: old players. Renin-angiotensin system. Kidney Int 2014;4:S58-64.

-12 Passos-Silva DG, Brandan E, Santos RA: Angiotensins as therapeutic targets beyond heart disease. Trends Pharmacol Sci 2015;36:310-320.

13 van Thiel BS, van der Pluijm I, te Riet L, Essers J, Danser AH: The renin-angiotensin system and its involvement in vascular disease. Eur J Pharmacol 2015;763:3-14.

14 An SJ, Liu P, Shao TM, Wang ZJ, Lu HG, Jiao Z, Li X, Fu JQ: Characterization and functions of vascular adventitial fibroblast subpopulations. Cell Physiol Biochem 2015;35:1137-1150.

15 Yang F, Chung AC, Huang XR, Lan HY: Angiotensin II induces connective tissue growth factor and collagen I expression via transforming growth factor-beta-dependent and -independent Smad pathways: the role of Smad3. Hypertension 2009;54:877-884.

16 Uhal BD, Li X, Piasecki CC, Molina-Molina M: Angiotensin signalling in pulmonary fibrosis. Int J Biochem Cell Biol 2012;44:465-468.

17 Dikalov SI, Nazarewicz RR: Angiotensin II-induced production of mitochondrial reactive oxygen species: potential mechanisms and relevance for cardiovascular disease. Antioxid Redox Signal 2013;19:10851094.

18 Toblli JE, Stella I, Mazza ON, Ferder L, Inserra F: Different effect of losartan and amlodipine on penile structures in male spontaneously hypertensive rats. Am J Nephrol 2004;24:614-623.

19 Kilarkaje N, Yousif MH, El-Hashim AZ, Makki B, Akhtar S, Benter IF: Role of angiotensin II and angiotensin-(1-7) in diabetes-induced oxidative DNA damage in the corpus cavernosum. Fertil Steril 2013;100:226-233.

20 Li WJ, Wang H, Zhou J, Li B, Zhang J, Lu M, Wang Z: P144, A TGF-beta1 antagonist peptide, synergizes with sildenafil and enhances erectile response via amelioration of cavernosal fibrosis in diabetic rats. J Sex Med 2013;10:2942-2951.

21 Canguven O, Lagoda G, Sezen SF, Burnett AL: Losartan preserves erectile function after bilateral cavernous nerve injury via antifibrotic mechanisms in male rats. J Urol 2009;181:2816-2822.

22 Daniel C: Blocking of angiotensin II is more than blocking of transforming growth factor-beta. Kidney Int 2008;74:551-553.

23 Toblli JE, Cao G, Lombrana A, Rivero M: Functional and morphological improvement in erectile tissue of hypertensive rats by long-term combined therapy with phosphodiesterase type 5 inhibitor and losartan. J Sex Med 2007;4:1291-1303.

24 Li WJ, Zhou J, Li B, Wang H, Peng YB, Wang Z: PARP inhibition restores erectile function by suppressing corporal smooth muscle apoptosis in diabetic rats. J Sex Med 2011;8:1072-1082. 


\section{Cellular Physiology Cell Physiol Biochem 2017;42:333-345

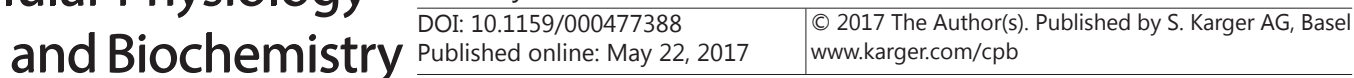

Li et al.: Losartan Preserves Erectile Function by Suppression of Fibrosis and CVOD

25 Lundberg JO, Weitzberg E: NO generation from nitrite and its role in vascular control. Arterioscler Thromb Vasc Biol 2005;25:915-922.

26 Davila HH, Rajfer J, Gonzalez-Cadavid NF: Corporal veno-occlusive dysfunction in aging rats: evaluation by cavernosometry and cavernosography. Urology 2004;64:1261-1266.

-27 Kifor I, Williams GH, Vickers MA, Sullivan MP, Jodbert P, Dluhy RG: Tissue angiotensin II as a modulator of erectile function. I. Angiotensin peptide content, secretion and effects in the corpus cavernosum. J Urol 1997;157:1920-1925.

28 Ertemi H, Lau DH, Mikhailidis DP, Mumtaz FH, Thompson CS: Angiotensin II increases corpus cavernosal contractility and oxidative stress in partial bladder outlet obstructed rabbits: relevance to erectile dysfunction. J Sex Med 2013;10:1251-1258.

-29 Yang R, Yang B, Wen Y, Fang F, Cui S, Lin G, Sun Z, Wang R, Dai Y: Losartan, an Angiotensin type I receptor, restores erectile function by downregulation of cavernous renin-angiotensin system in streptozocininduced diabetic rats. J Sex Med 2009;6:696-707.

30 Chen Y, Cui S, Lin H, Xu Z, Zhu W, Shi L, Yang R, Wang R, Dai Y: Losartan improves erectile dysfunction in diabetic patients: a clinical trial. Int J Impot Res 2012;24:217-220.

- 31 Abdel Aziz MT, El Asmer MF, Mostafa T, Atta H, Mahfouz S, Fouad H, Rashed L, Sabry D, Hassouna A, Abdel Aziz AT, Senbel A, Demery A: Effects of losartan, HO-1 inducers or HO-1 inhibitors on erectile signaling in diabetic rats. J Sex Med 2009;6:3254-3264.

-32 Kovanecz I, Ferrini MG, Vernet D, Nolazco G, Rajfer J, Gonzalez-Cadavid NF: Pioglitazone prevents corporal veno-occlusive dysfunction in a rat model of type 2 diabetes mellitus. BJU Int 2006;98:116-124.

-33 Chung WS, Shim BS, Park YY: Hemodynamic insult by vascular risk factors and pharmacologic erection in men with erectile dysfunction: Doppler sonography study. World J Urol 2000;18:427-430.

-34 Park K, Ryu KS, Li WJ, Kim SW, Paick JS: Chronic treatment with a type 5 phosphodiesterase inhibitor suppresses apoptosis of corporal smooth muscle by potentiating Akt signalling in a rat model of diabetic erectile dysfunction. Eur Urol 2008;53:1282-1288.

- 35 Ahn GJ, Sohn YS, Kang KK, Ahn BO, Kwon JW, Kang SK, Lee BC, Hwang WS: The effect of PDE5 inhibition on the erectile function in streptozotocin-induced diabetic rats. Int J Impot Res 2005;17:134-141.

-36 Mostafa ME, Senbel AM, Mostafa T: Effect of chronic low-dose tadalafil on penile cavernous tissues in diabetic rats. Urology 2013;81:1253-1259.

37 Lombo C, Morgado C, Tavares I, Neves D: Effects of prolonged ingestion of epigallocatechin gallate on diabetes type 1-induced vascular modifications in the erectile tissue of rats. Int J Impot Res 2016;28:133138.

38 Duffield JS, Lupher M, Thannickal VJ, Wynn TA: Host responses in tissue repair and fibrosis. Annu Rev Pathol 2013;8:241-276.

-39 Abdalla M, Goc A, Segar L, Somanath PR: Akt1 mediates alpha-smooth muscle actin expression and myofibroblast differentiation via myocardin and serum response factor. J Biol Chem 2013;288:3348333493.

40 Ding G, Reddy K, Kapasi AA, Franki N, Gibbons N, Kasinath BS, Singhal PC: Angiotensin II induces apoptosis in rat glomerular epithelial cells. Am J Physiol Renal Physiol 2002;283:F173-180.

-41 Lee YH, Mungunsukh 0, Tutino RL, Marquez AP, Day RM: Angiotensin-II-induced apoptosis requires regulation of nucleolin and Bcl-xL by SHP-2 in primary lung endothelial cells. J Cell Sci 2010;123:16341643.

42 Zhang L, Ren Z, Yang Q Ding G: Csk regulates angiotensin II-induced podocyte apoptosis. Apoptosis 2016;21:846-855.

43 Friedberg MK, Cho MY, Li J, Assad RS, Sun M, Rohailla S, Honjo O, Apitz C, Redington AN: Adverse biventricular remodeling in isolated right ventricular hypertension is mediated by increased transforming growth factor-beta1 signaling and is abrogated by angiotensin receptor blockade. Am J Respir Cell Mol Biol 2013;49:1019-1028.

44 Montezano AC, Nguyen Dinh Cat A, Rios FJ, Touyz RM: Angiotensin II and vascular injury.Curr Hypertens Rep 2014;16:431.

45 Li WJ, Park K, Paick JS, Kim SW: Chronic treatment with an oral rho-kinase inhibitor restores erectile function by suppressing corporal apoptosis in diabetic rats. J Sex Med 2011;8:400-410.

-46 Jin L, Burnett AL: RhoA/Rho-kinase in erectile tissue: mechanisms of disease and therapeutic insights. Clin Sci (Lond) 2006;110:153-165. 